\title{
Resummation of Quantum Radiation Reaction in Plane Waves
}

\author{
Greger Torgrimsson $\oplus^{*}$ \\ Helmholtz-Zentrum Dresden-Rossendorf, Bautzner Landstraße 400, 01328 Dresden, Germany
}

(Received 4 March 2021; revised 3 June 2021; accepted 6 August 2021; published 9 September 2021)

\begin{abstract}
We propose a new approach to obtain the momentum expectation value of an electron in a high-intensity laser, including multiple photon emissions and loops. We find a recursive formula that allows us to obtain the $\mathcal{O}\left(\alpha^{n}\right)$ term from $\mathcal{O}\left(\alpha^{n-1}\right)$, which can also be expressed as an integro-differential equation. In the classical limit we obtain the solution to the Landau-Lifshitz equation to all orders. We show how spindependent quantum radiation reaction can be obtained by resumming both the energy expansion as well as the $\alpha$ expansion.
\end{abstract}

DOI: 10.1103/PhysRevLett.127.111602

An electron in an electromagnetic field emits photons and the recoil it experiences is called radiation reaction (RR) [1,2]. Classically, the standard equation is the Abraham-Lorentz-Dirac (LAD) equation, but since it leads to unphysical solutions (with either preacceleration, where the particle starts to accelerate before the field turns on, or runaways, where the particle's momentum diverges even in the absence of a field) it is common to replace it with the Landau-Lifshitz (LL) equation, which is free from such unphysical solutions. Since preacceleration occurs on timescales that are actually within the quantum regime, and since the LAD and LL equations agree quite well within the classical regime (see, e.g., [3]), it is common to view the LL equation as giving a correct description for practical purposes. A more practical problem is how to describe quantum RR. If RR is important one would in general expect the particle to emit many photons, making higher orders in $\alpha=e^{2} /(4 \pi)$ important. The first RR experiments with high-intensity lasers were performed recently [4,5] (see also [6]), and in upcoming experiments one may expect significant quantum effects [7-11]. Clearly, one needs some approximation to obtain higher orders in $\alpha$. Standard approaches assume [12] $a_{0}:=E / \omega \gg 1$ and then approximate higher orders as incoherent products [13] of $\mathcal{O}(\alpha)$ photon emissions calculated with a locally constant field (see, e.g., [7-11,24]). A question is how to take spin into account. See, e.g., [25-27] for spin-dependent RR. In this Letter we propose a new method for obtaining quantum $\mathrm{RR}$ in high-intensity lasers. It too is based on incoherent products of $\mathcal{O}(\alpha)$ terms. The main differences compared to previous approaches are: (1) We treat spin transitions using

Published by the American Physical Society under the terms of the Creative Commons Attribution 4.0 International license. Further distribution of this work must maintain attribution to the author(s) and the published article's title, journal citation, and DOI. Funded by SCOAP ${ }^{3}$.
Mueller matrices, which allows us to consider arbitrary spin, field polarization and field shape, and, importantly, we are not limited to large $a_{0}$. (2) We consider the contribution from loop diagrams explicitly. (3) While we can resum the $\alpha$ expansion right from the start into a new integro-differential equation, we also show that resummation methods based, e.g., on Padé approximants can be faster.

Let $p_{\mu}$ and $P_{\mu}$ be the initial and final momentum of the electron, $k_{\mu}$ the wave vector of the plane wave, which is described by a gauge potential $a_{\perp}(\sigma)$ where [12] $\sigma=k x=$ $\omega(t+z)$ is the (rescaled) light-front time and $a_{ \pm}=0$. Light-front coordinates are defined by $v^{ \pm}=2 v_{\mp}=v^{0} \pm v^{3}$ and $v_{\perp}=\left\{v_{1}, v_{2}\right\}$. We will focus on $\langle k P\rangle=\sum_{n=0}^{\infty}\langle k P\rangle^{(n)}$, where $\langle k P\rangle^{(n)}=\mathcal{O}\left(\alpha^{n}\right)$. The reason for focusing on the light-front longitudinal momentum $\left(k P=2 k_{+} P_{-}\right)$is because in a plane wave the probabilities of the whole process and each intermediate step only depend nontrivially on this momentum component. The dependence on the initial and final spin can be expressed as $\langle k P\rangle^{(n)}=(1 / 2) \mathbf{N}_{0} \cdot \mathbf{M}^{(n)} \cdot \mathbf{N}_{f}$, where $\mathbf{N}=\{1, \mathbf{n}\}$ is the Stokes vector for spin along the unit vector $\mathbf{n}$ and $\mathbf{M}^{(n)}$ are "strong-field-QED Mueller matrices" [21,28,29]. Averaging and summing over the initial and final spin gives $\{1, \mathbf{0}\} \cdot \mathbf{M} \cdot\{1, \mathbf{0}\}$. The Mueller-matrix approach has been developed recently $[21,28,29]$. It allows us to obtain $\mathcal{O}\left(\alpha^{n}\right)$ probabilities to leading order for long pulses or large $a_{0}=E / \omega$ by multiplying $\mathcal{O}(\alpha)$ Mueller matrices. In this case we need $\mathbf{M}^{C}$ for (nonlinear) Compton scattering and $\mathbf{M}^{L}$ for the cross term between the $\mathcal{O}\left(\alpha^{0}\right)$ and $\mathcal{O}(\alpha)$ parts of the amplitude for $e^{-} \rightarrow e^{-}$. In $[21,28,29]$ we described how to write down expressions for individual higher-order diagrams. To use these methods to obtain RR we need a way to evaluate all the relevant higher-order diagrams and to resum them. Both photon emissions and loops [30] contribute and there are $2^{n}$ diagrams at $\mathcal{O}\left(\alpha^{n}\right)$. More details of this derivation can be found in the Supplemental 
Material (SM) [35]. One of the main steps is to note that $\mathbf{M}^{(n)}$ can be obtained by prepending a Compton scattering or a loop step at the initial position of $\mathbf{M}^{(n-1)}$, which gives the following recursive formula:

$$
\begin{aligned}
\mathbf{M}^{(n)}\left(b_{0}, \sigma\right)= & \int_{\sigma}^{\infty} \frac{d \sigma^{\prime}}{b_{0}} \int_{0}^{1} d q\left\{\mathbf{M}^{L}\left(b_{0}, \sigma^{\prime}, q\right) \cdot \mathbf{M}^{(n-1)}\left(b_{0}, \sigma^{\prime}\right)\right. \\
& \left.+\mathbf{M}^{C}\left(b_{0}, \sigma^{\prime}, q\right) \cdot \mathbf{M}^{(n-1)}\left(b_{0}[1-q], \sigma^{\prime}\right)\right\},
\end{aligned}
$$

where $b_{0}=k p, q=k l / k p$ and $l$ is the photon momentum, and $\mathbf{M}^{(0)}=b_{0} \mathbf{1}\left[\mathbf{M}^{(0)}=\mathbf{1}\right.$ for the probability]. The lower integration limit has been introduced so that the products of Mueller matrices are light-front-time ordered. The final result is obtained by setting $\sigma=-\infty$. The shift $b_{0} \rightarrow$ $b_{0}\left(1-q_{1}\right)$ in the Compton term takes RR into account.

With $\mathbf{M}=\sum_{n=0}^{\infty} \mathbf{M}^{(n)}$, (1) implies

$\frac{\partial \mathbf{M}}{\partial \sigma}=-\int_{0}^{1} \frac{d q}{b_{0}}\left\{\mathbf{M}^{L} \cdot \mathbf{M}\left(b_{0}\right)+\mathbf{M}^{C} \cdot \mathbf{M}\left(b_{0}[1-q]\right)\right\}$.

This integro-differential matrix equation gives quantum RR to all orders in $\alpha$ with spin taken into account. Note that even if we are only interested in unpolarized initial and final states, we still need to solve a matrix equation before we can project with $\{1, \mathbf{0}\} \cdot \mathbf{M} \cdot\{1, \mathbf{0}\}$. Note also that (2) holds even if $a_{0}$ is not large, provided the field is sufficiently long and the full version of $\mathbf{M}^{C, L}$ is used. Equation (2) looks similar to kinetic RR equations [10,36] (see also [37]), so one may expect that it can be solved with similar methods. However, in this Letter we will instead use (1) to obtain the first $\mathbf{M}^{(n)}$ terms before performing a resummation. It turns out that this resummation approach can be much faster.

We consider first the classical limit. In the SM we show that (1) leads to a geometric series in the classical limit. In fact, we also show that the $\left\langle P_{\perp,+}\right\rangle$ components can be calculated in a similar way. We find

$\lim _{b_{0} \ll 1}\left\langle P_{-, \perp}\right\rangle=\pi_{-, \perp}+\frac{\Delta}{1+\Delta \int d \sigma \mathbf{a}^{\prime 2}}\left[\pi^{\prime}-\int d \sigma \mathbf{a}^{\prime 2} \pi\right]_{-, \perp}$,

where $\pi_{\mu}=p_{\mu}-a_{\mu}+\left(2 a p-a^{2}\right) k_{\mu} /(2 k p)$ is the Lorentz momentum, and $\left\langle P_{+}\right\rangle$satisfies the mass-shell condition $P_{+}=\left(P_{\perp}^{2}+1\right) /\left(4 P_{-}\right)$. This resummation [38] agrees exactly with the exact solution to LL [40] (see also [41] for an exact solution to LL; $[10,36,42]$ for comparisons between LL and kinetic equations; and [43-47] for comparisons between classical equations and the classical limit of quantum RR at first order). Note that the loops are needed even in the classical limit, cf. [46-48].

This does not mean that LAD does not agree with the classical limit of QED, because our approximation neglects terms that have subdominant scaling with respect to the pulse length and/or $a_{0}$. From Eq. (4.39) in [47] we see that the difference between the LL and LAD equations at $\mathcal{O}\left(\alpha^{2}\right)$ is a term proportional to $\mathbf{a}^{\prime 2}$, i.e., a term without an integral. This term vanishes at asymptotic $\sigma$, but is also subdominant at finite times because it has no pulse-length scaling. We can therefore not rule out that the classical limit of all QED contributions may agree with the LAD equation rather than the LL equation.

In the limit of a very long pulse we have (cf. [3,49-51])

$$
\left\langle P_{-}\right\rangle \approx \frac{p_{-}}{\Delta \int d \sigma^{\prime} \mathbf{a}^{\prime 2}}\left\langle P_{\perp}\right\rangle \approx \frac{\int d \sigma^{\prime} \mathbf{a}^{\prime 2}\left[a\left(\sigma^{\prime}\right)-a(\sigma)\right]_{\perp}}{\int d \sigma^{\prime} \mathbf{a}^{\prime 2}},
$$

so $\left\langle P_{-}\right\rangle$becomes small, $\left\langle P_{\perp}\right\rangle$ stays $\mathcal{O}(1)$ and $\left\langle P_{+}\right\rangle$ becomes large, and (since $\Delta \propto p_{-}$) all components of $\left\langle P_{\mu}\right\rangle$ become independent of the initial momentum [3,49-51]. This observation will be important for resumming the $\alpha$ expansion in the quantum regime.

Having shown that (1) gives the expected classical limit, we now turn to quantum RR. For simplicity we focus on $\left\langle P_{-}\right\rangle$, and we sum over the final spin, i.e., $\mathbf{N}_{f}=\{1, \mathbf{0}\}$ is fixed and we have an overall factor of 2 , so we use $\mathbf{N}^{(n)}:=\mathbf{M}^{(n)} \cdot\{1, \mathbf{0}\}$. In this first application we consider a constant field, so the $\sigma$ integrals give $\int d \sigma_{n} \ldots d \sigma_{1} \rightarrow$ $(\Delta \phi)^{n} / n$ !, and it is convenient to rescale $\mathbf{N}^{(n)}$ so that

$$
a_{0}\langle k P\rangle^{(n)}=T^{n} \mathbf{N}_{0} \cdot \mathbf{N}^{(n)},
$$

where $T=\alpha a_{0} \Delta \phi$ is our effective expansion parameter, which can be $T>1$ for large $a_{0} \Delta \phi$. In general $\mathbf{N}$ has four elements, but here we consider only initial and final states that are either unpolarized or polarized (anti-)parallel to the magnetic field, and then only two elements contribute. Omitting the irrelevant elements we have $\mathbf{N}_{0}=\{1,0\}$ and $\mathbf{N}_{0}=\{1, \pm 1\}$ for unpolarized and polarized states. We have

$$
\mathbf{N}^{(n)}=\int_{0}^{1} \frac{d q}{n \chi}\left\{\mathbf{M}^{C} \cdot \mathbf{N}^{(n-1)}(\chi[1-q])+\mathbf{M}^{L} \cdot \mathbf{N}^{(n-1)}(\chi)\right\},
$$

where $\mathbf{N}^{(0)}(\chi)=\chi\{1,0\}, 1 / n$ comes from $(\Delta \phi)^{n} / n !, \chi=$ $a_{0} b_{0}$ is the quantum nonlinearity parameter (gives the electric field in the rest frame of the electron), from [28,29]

$$
\mathbf{M}^{C}=\left(\begin{array}{cc}
-\mathrm{Ai}_{1}(\xi)-\kappa \frac{\mathrm{Ai}^{\prime}(\xi)}{\xi} & \frac{q}{s_{1}} \frac{\mathrm{Ai}(\xi)}{\sqrt{\xi}} \\
q \frac{\operatorname{Ai}(\xi)}{\sqrt{\xi}} & -\mathrm{Ai}_{1}(\xi)-2 \frac{\operatorname{Ai}^{\prime}(\xi)}{\xi}
\end{array}\right)
$$

and

$$
\mathbf{M}^{L}=\left(\begin{array}{cc}
\mathrm{Ai}_{1}(\xi)+\kappa \frac{\mathrm{Ai}^{\prime}(\xi)}{\xi} & -q \frac{\mathrm{Ai}(\xi)}{\sqrt{\xi}} \\
-q \frac{\mathrm{Ai}(\xi)}{\sqrt{\xi}} & \mathrm{Ai}_{1}(\xi)+\kappa \frac{\mathrm{Ai}^{\prime}(\xi)}{\xi}
\end{array}\right)
$$




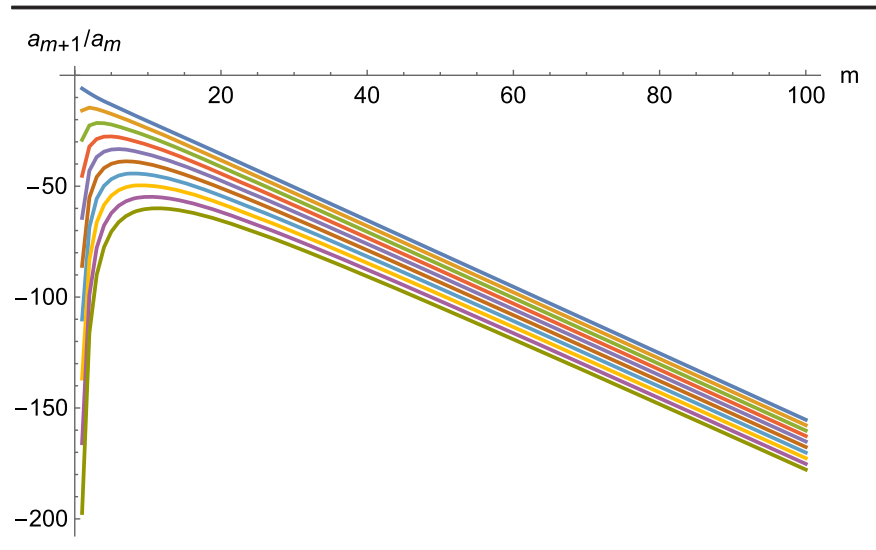

FIG. 1. The ratios of neighboring coefficients in the $\chi$ expansion for $\{1,0\} \cdot \mathbf{a}_{m}$. The different lines correspond to $\langle k P\rangle^{(1)}$ (top) to $\langle k P\rangle^{(10)}$ (bottom). The linear scaling at higher orders shows that the coefficients grow factorially and the $\chi$ expansion is hence asymptotic.

where $\xi=(r / \chi)^{2 / 3}$ with $r=\left(1 / s_{1}\right)-1, \kappa=\left(1 / s_{1}\right)+s_{1}$, $s_{1}=1-q$ and $\operatorname{Ai}_{1}(\xi)=\int_{\xi}^{\infty} d x \operatorname{Ai}(x)$.

In order to compute $\mathbf{N}^{(n)}$ we have used two completely different methods. In the first we compute $\mathbf{N}^{(1)}(\chi)$ between $\chi=0$ and some [52] $\chi_{\max }$ and make an interpolation function of it, which is then used in (6) to compute an interpolation function of $\mathbf{N}^{(2)}(\chi)$, and so on.

In the second method we expand $\mathbf{N}^{(1)}(\chi)$ in a power series in $\chi$, which is then used to obtain a power series of $\mathbf{N}^{(2)}(\chi)$ and so on, see the SM. This gives $\mathbf{N}^{(n)}=\chi^{1+n} \sum_{m=0}^{M} \mathbf{a}_{m}^{(n)} \chi^{m}$. As illustrated in Fig. 1, the coefficients have alternating sign and grow factorially $\mathbf{a}_{m} \sim(-1)^{m} m$ !. The $\chi$ expansion is therefore asymptotic with zero radius of convergence and needs to be resummed. There is no unique resummation method (unless, of course, one is able to find an exact expression). Different resummations are obtained by matching the series onto different (sums of) special functions. Recent examples involve, e.g., the Meijer-G or hypergeometric functions [23,53,54] (see [55] for further applications in strong-field QED). Such resummations require fewer terms, but usually some extra information, e.g., about the opposite limit (large $\chi$ in our case). However, in the present case, we can without problems obtain a large number of terms quickly. We can hence use the general Borel-Padé resummation method [56-65], which only requires the $\mathbf{a}_{m}^{(n)}$ coefficients. From the truncated series one first obtains a truncated Borel transform, $\mathbf{B}_{M}^{(n)}(t)=\sum_{m=0}^{M}(1 / m !) \mathbf{a}_{m}^{(n)} t^{m}$. Next we project with the initial Stokes vector, $a_{m}^{(n)}:=\mathbf{N}_{0} \cdot \mathbf{a}_{m}^{(n)}$, and construct a Padé approximant $P B[I / J](t)=$ $\sum_{i=0}^{I} c_{i} t^{i} /\left(1+\sum_{j=1}^{J} d_{j} t^{j}\right)=B_{N}(t)+\mathcal{O}\left(t^{N+1}\right)$. The result is then obtained from the following Laplace integral:

$$
\mathbf{N}_{0} \cdot \mathbf{N}_{\mathrm{re}}^{(n)}(\chi)=\chi^{1+n} \int_{0}^{\infty} \frac{d t}{\chi} e^{-t / \chi} P B[I / J](t) .
$$

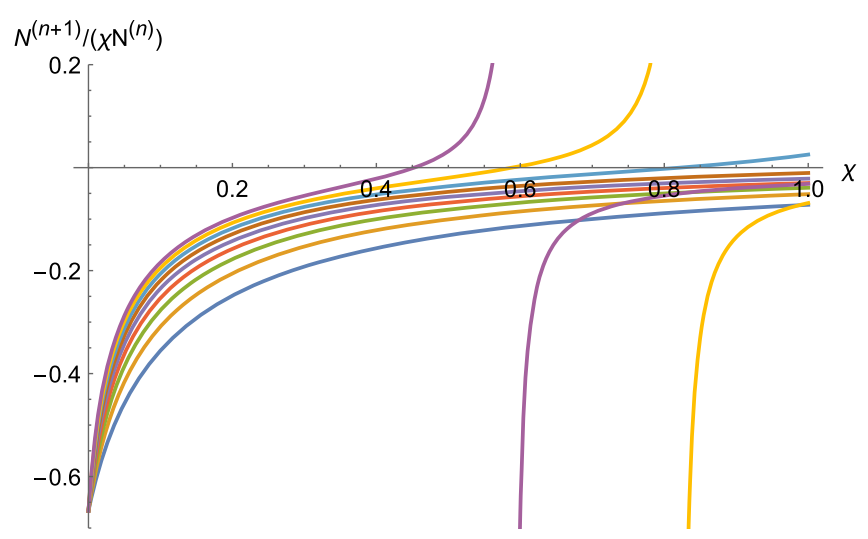

FIG. 2. The ratios $N^{(n+1)} /\left(\chi N^{(n)}\right)$ of neighboring coefficients in the $\alpha$ expansion as a function of $\chi$, for an unpolarized initial state, $N^{(n)}=\{1,0\} \cdot \mathbf{N}^{(n)}$. The ratios have been divided by $\chi$ in order to show the convergence to the classical limit $N^{(n+1)} / N^{(n)} \rightarrow-2 \chi / 3$ for $\chi \rightarrow 0$.

Using either of these two approaches we obtain a set of functions, $\mathbf{N}^{(n)}(\chi)$, for $0<\chi<\chi_{\max }$. The result for an unpolarized initial state is shown in Fig. 2. In the low $\chi$ limit we find convergence towards the classical prediction.

In general one would also expect the $\alpha$ expansion to be asymptotic. However, an approximation does not have be asymptotic, see, e.g., [60,66-69] for the weak and strong field approximations of the QED effective action. (See [70,71] for other recent $\alpha$ resummations.) In classical RR, LAD leads to an asymptotic series [72], while LL has a finite radius of convergence. Since the coefficients we have calculated suggest a finite radius of convergence, we propose to resum the $\alpha$ expansion with a Padé approximant

$$
a_{0}\langle k P\rangle \approx a_{0}\langle k P\rangle_{\mathrm{re}}=\chi+\frac{\sum_{i=1}^{I} A_{i}(\chi) T^{i}}{1+\sum_{j=1}^{J} B_{j}(\chi) T^{j}},
$$

where $A_{i}$ and $B_{i}$ are obtained by matching $a_{0}\langle k P\rangle_{\mathrm{re}}=$ $\chi+\sum_{n=0}^{I+J} T^{n} \mathbf{N}_{0} \cdot \mathbf{N}^{(n)}+\mathcal{O}\left(T^{I+J+1}\right)$. Since we expect a finite limit for $T \gg 1$, we take $I=J$. This makes it possible for $\langle k P\rangle_{\text {re }} \rightarrow 0$ as $T \rightarrow \infty$, which is what we expect [cf. (4)]. If we do not impose this limit, then we can take $\left|A_{I} /\left(\chi B_{I}\right)+1\right|$ as an upper-limit estimate on the relative error (the error goes to zero as $T \rightarrow 0$ ). Alternatively, we can demand $\langle k P\rangle_{\mathrm{re}}=\mathcal{O}(1 / T)$ for $T \gg 1$, which fixes $A_{I}=-\chi B_{I}$. LL predicts that the leading order in $T \gg 1$ is independent of the initial momentum (4). If we assume that holds in general, then the $\mathcal{O}(1 / T)$ term must be the same as the classical prediction, which implies

$$
A_{I}=-\chi B_{I} \quad B_{I}=\frac{2}{3}\left(A_{I-1}+\chi B_{I-1}\right) .
$$

Figure 3 shows that the resummation (10) converges quickly. On the scale of this plot, the $I=4$ and $I=5$ resummations are virtually indistinguishable, which are 


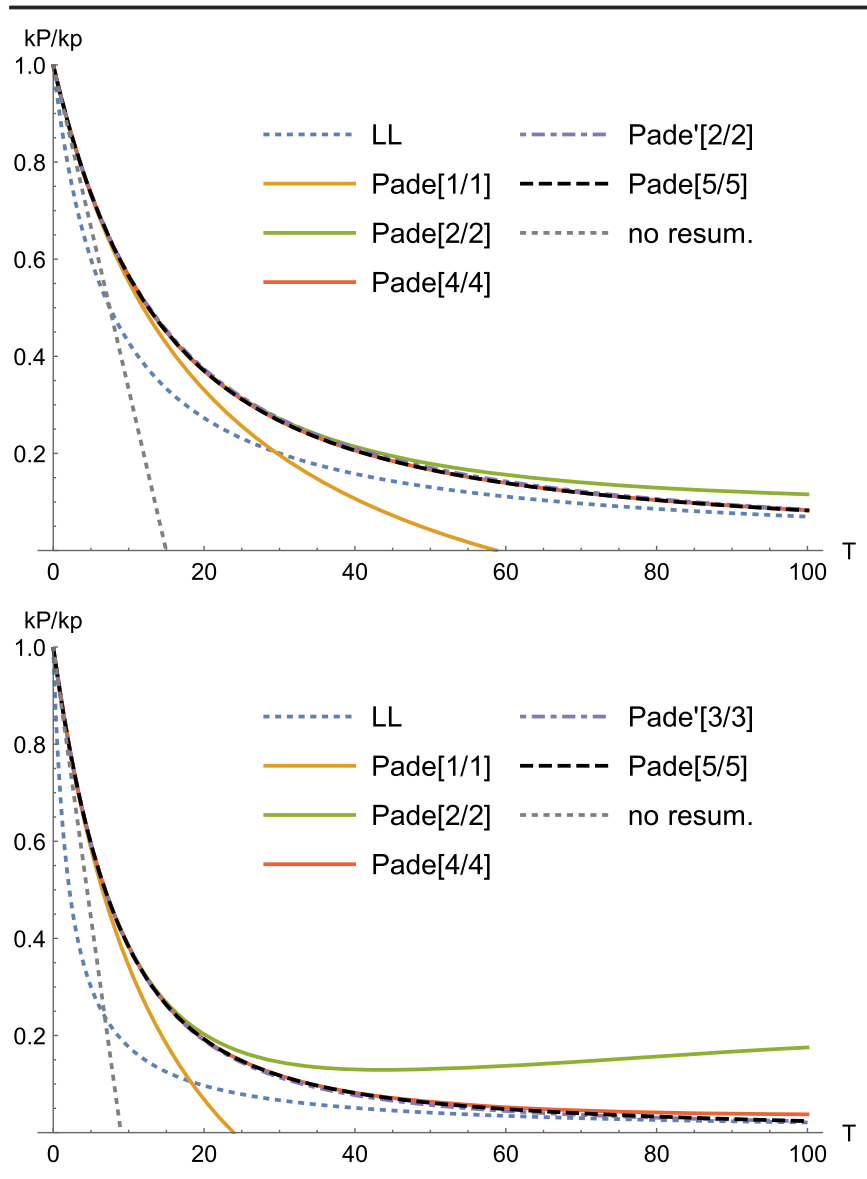

FIG. 3. Final result for $\chi=0.2$ (upper) and $\chi=0.7$ (lower) as a function of $T=\alpha a_{0} \Delta \phi$. Padé $[I / I]$ corresponds to the resummation in (10) with $A_{i}$ and $B_{i}$ determined from the first $2 I$ coefficients $\{1,0\} \cdot \mathbf{N}^{(n)}, 1 \leq n \leq 2 I$. Padé' $[I / I]$ is obtained from only $\{1,0\} \cdot \mathbf{N}^{(n)}, 1 \leq n \leq 2 I-2$, while the remaining two coefficients are determined from (11). The "no resum." line is just the sum of $\mathcal{O}\left(\alpha^{0}\right)$ and $\mathcal{O}(\alpha)$.

obtained from the first eight and ten $\mathbf{N}^{(n)}$ terms. For short to moderately large $T$, we see that the classical prediction overestimates the effect of RR, as expected [1]. However, for larger $T$ the classical and quantum predictions seem to converge. This is what one would expect if the leading order at $T \gg 1$ is independent of the initial momentum. This motivates us to use the modified Padé approximant based on (11). With the two extra conditions in (11) we indeed find an even faster convergence, with a decent approximation already with $I=2$, i.e., using only the $\mathcal{O}(\alpha)$ and $\mathcal{O}\left(\alpha^{2}\right)$ terms.

These resummations can be compared with the solution to the integro-differential equation corresponding to (6), i.e.,

$$
\frac{\partial \mathbf{N}}{\partial T}=\int_{0}^{1} \frac{d q}{\chi}\left\{\mathbf{M}^{C} \cdot \mathbf{N}(\chi[1-q])+\mathbf{M}^{L} \cdot \mathbf{N}(\chi)\right\},
$$

where $\mathbf{N}=\sum_{n=0}^{\infty} T^{n} \mathbf{N}^{(n)}$ and $\mathbf{N}(T=0)=\chi\{1,0\}$. We have solved (12) numerically with the Euler method and

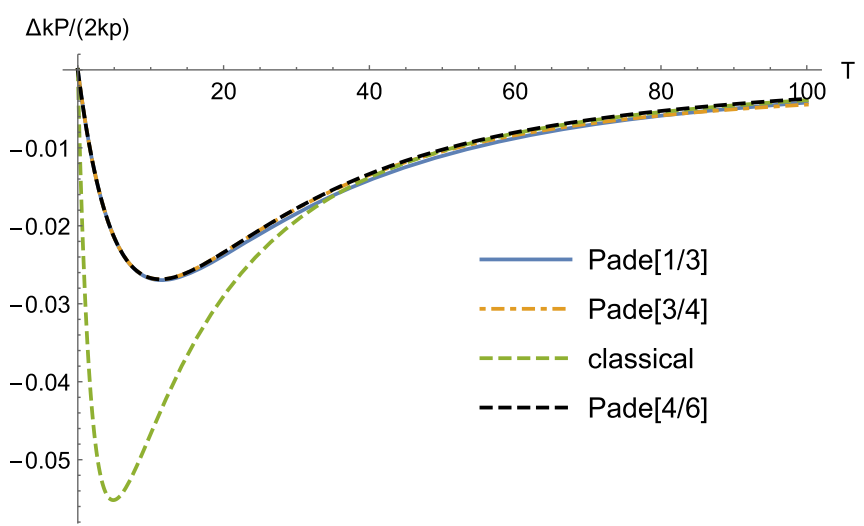

FIG. 4. Difference in the final momentum for initial state with spin up and down and $\chi=0.2$.

found good agreement with the resummations above. However, it takes much more time to solve (12) because we need $T_{\max } / d T$ steps, which is typically more than the ten (or fewer) steps we needed in the resummation approach.

At $T \gg 1$ we have the ansatz $\mathbf{N} \approx\left\{c / T+\mathcal{O}\left(1 / T^{2}\right)\right.$, $\left.\mathcal{O}\left(1 / T^{2}\right)\right\}$, so $\partial \mathbf{N} / \partial T=\mathcal{O}\left(1 / T^{2}\right)$, which gives a condition for $c$ since the right-hand side of (12) is not automatically $\mathcal{O}\left(1 / T^{2}\right)$. As mentioned, we expect $c$ to be independent of $\chi$, and now we can confirm that this is consistent with (12). In order to obtain $\mathbf{N}^{(n)}$ from $\mathbf{N}^{(n-1)}$ we need to calculate both components of $\mathbf{N}^{(n-1)}$, even if we at the end are only interested in unpolarized particles. Hence, in calculating the results above we have obtained the necessary information to study a polarized initial state as a byproduct. We show in the SM for the difference in the final momentum due to the initial spin

$\lim _{\chi \ll 1} \frac{a_{0}}{2} k \Delta\langle P\rangle=\sum_{n=1}^{\infty}\{0,1\} \cdot \mathbf{N}^{(n)} T^{n}=-\frac{3}{2} \chi^{2} \frac{\ln \left[1+\frac{2}{3} \chi T\right]}{\left(1+\frac{2}{3} \chi T\right)^{2}}$.

We see that $\Delta\langle P\rangle$ too becomes independent of $\chi$ to leading order in $T \gg 1$, although this time the next-to-leading order is only logarithmically suppressed. Another difference is that (13) is nonmonotonic with a maximum $|\Delta\langle P\rangle|$ at $T \sim 1 / \chi$.

The full quantum result can be obtained as described above. Although (13) might suggest using a resummation involving logarithms, we still find a fast convergence with Padé approximants as in (10), except that $P_{-}>0$ implies that $|\Delta\langle P\rangle|$ must be smaller than $\langle P\rangle_{\uparrow}+\langle P\rangle_{\downarrow}$ and for that to hold at large $T$ we need $J>I$. In practice, different choices of $I$ and $J$ can give good approximations, and a "wrong" choice just means that we need to include more terms. Figure 4 shows that the classical prediction overestimates the peak of $|\Delta\langle P\rangle|$ by about a factor of 2 for $\chi=0.2$, but is close to the quantum result for large $T$. 
In conclusion, we have developed a new approach for spin-dependent quantum RR using products of Mueller matrices for photon emissions and loops. We have found a recursive formula which gives $\mathcal{O}\left(\alpha^{n}\right)$ from $\mathcal{O}\left(\alpha^{n-1}\right)$. In the classical limit we find the solution to LL to all orders. We obtain quantum RR either by resumming the recursive formula into an integro-differential matrix equation, or by resumming the $\alpha$ expansion with Padé approximants, which converge quickly. The $\mathcal{O}\left(\alpha^{n}\right)$ terms in the second approach can be obtained either numerically or by expanding each $\mathcal{O}\left(\alpha^{n}\right)$ in $\chi$ and resumming the resulting asymptotic series with, e.g., the Borel-Padé method.

The first laser-based RR experiments were performed just a couple of years ago $[4,5]$, and further laser-electron experiments are planned for the near future, e.g., at LUXE [73,74] and FACET-II [75]. The experiment in [5] has already hinted that standard locally-constant-field (LCF) approaches might give significant discrepancies. One source of discrepancy within LCF could be spin and loop effects not taken into account in standard LCF approaches, but which can be included in a LCF version of our approach. If instead $a_{0}$ is not large enough for any LCF treatment, but if the field is long, then one can try our approach with a locally-monochromatic-field (LMF) approximation. If $a_{0}$ is not large enough for LCF and the pulse not long enough for LMF, but if $a_{0}$ is still moderately large and the pulse still moderately long, then one could try our approach with the exact $\mathcal{O}(\alpha)$ Mueller matrices $[28,29]$, which would also be relevant for long pulses with some asymmetry that prevents a LMF treatment. In order to compare with experiments such as [5], it would be useful to generalize our approach to the momentum spectrum.

*g.torgrimsson@hzdr.de

[1] D. A. Burton and A. Noble, Aspects of electromagnetic radiation reaction in strong fields, Contemp. Phys. 55, 110 (2014).

[2] T. G. Blackburn, Radiation reaction in electron-beam interactions with high-intensity lasers, Rev. Mod. Plasma Phys. 4, 5 (2020).

[3] S. V. Bulanov, T. Zh. Esirkepov, M. Kando, J. K. Koga, and S. S. Bulanov, Lorentz-Abraham-Dirac versus LandauLifshitz radiation friction force in the ultrarelativistic electron interaction with electromagnetic wave (exact solutions), Phys. Rev. E 84, 056605 (2011).

[4] J. M. Cole, K. T. Behm, E. Gerstmayr, T. G. Blackburn, J. C. Wood, C. D. Baird, M. J. Duff, C. Harvey, A. Ilderton, A. S. Joglekar et al. Experimental Evidence of Radiation Reaction in the Collision of a High-Intensity Laser Pulse with a Laser-Wakefield Accelerated Electron Beam, Phys. Rev. X 8, 011020 (2018).

[5] K. Poder, M. Tamburini, G. Sarri, A. Di Piazza, S. Kuschel, C. D. Baird, K. Behm, S. Bohlen, J. M. Cole, and D. J. Corvan et al., Experimental Signatures of the Quantum
Nature of Radiation Reaction in the Field of an Ultraintense Laser, Phys. Rev. X 8, 031004 (2018).

[6] T. N. Wistisen, A. Di Piazza, H. V. Knudsen, and U. I. Uggerhøj, Experimental evidence of quantum radiation reaction in aligned crystals, Nat. Commun. 9, 795 (2018).

[7] T. G. Blackburn, C. P. Ridgers, J. G. Kirk, and A. R. Bell, Quantum Radiation Reaction in Laser-Electron Beam Collisions, Phys. Rev. Lett. 112, 015001 (2014).

[8] M. Vranic, T. Grismayer, R. A. Fonseca, and L. O. Silva, Quantum radiation reaction in head-on laser electron beam interaction, New J. Phys. 18, 073035 (2016).

[9] V. Dinu, C. Harvey, A. Ilderton, M. Marklund, and G. Torgrimsson, Quantum Radiation Reaction: From Interference to Incoherence, Phys. Rev. Lett. 116, 044801 (2016).

[10] N. Neitz and A. Di Piazza, Stochasticity Effects in Quantum Radiation Reaction, Phys. Rev. Lett. 111, 054802 (2013).

[11] S. R. Yoffe, Y. Kravets, A. Noble, and D. A. Jaroszynski, Longitudinal and transverse cooling of relativistic electron beams in intense laser pulses, New J. Phys. 17, 053025 (2015)

[12] We use units with $\hbar=c=m_{e}=1$, where $m_{e}$ is the electron mass. $E$ is the field strength and $\omega$ a characteristic frequency scale. We absorb the electron charge into the background field, $e E \rightarrow E$. Lorentz scalar products are denoted as $k p=k_{\mu} p^{\mu}=k_{0} p_{0}-k_{i} p_{i}$.

[13] Corrections to the incoherent-product approximation have been studied in detail for $\mathcal{O}\left(\alpha^{2}\right)$ processes in Refs. [14-23].

[14] V. N. Baier, V. M. Katkov, and V. M. Strakhovenko, Higherorder effects in external field: Pair production by a particle, Sov. J. Nucl. Phys. 14, 572 (1972).

[15] V. I. Ritus, Vacuum polarization correction to elastic electron and muon scattering in an intense field and pair electroand muoproduction, Nucl. Phys. B44, 236 (1972).

[16] B. King and H. Ruhl, Trident pair production in a constant crossed field, Phys. Rev. D 88, 013005 (2013).

[17] V. Dinu and G. Torgrimsson, Trident pair production in plane waves: Coherence, exchange, and spacetime inhomogeneity, Phys. Rev. D 97, 036021 (2018).

[18] B. King and A. M. Fedotov, Effect of interference on the trident process in a constant crossed field, Phys. Rev. D 98, 016005 (2018).

[19] D. A. Morozov and V. I. Ritus, Elastic electron scattering in an intense field and two-photon emission, Nucl. Phys. B86, 309 (1975).

[20] B. King, Double Compton scattering in a constant crossed field, Phys. Rev. A 91, 033415 (2015).

[21] V. Dinu and G. Torgrimsson, Single and double nonlinear Compton scattering, Phys. Rev. D 99, 096018 (2019).

[22] D. A. Morozov and N. B. Narozhnyi, Elastic scattering of photons in an intense field and the photoproduction of a pair and a photon, Sov. Phys. JETP 45, 23 (1977), http://jetp.ras .ru/cgi-bin/e/index/e/45/1/p23?a=list.

[23] G. Torgrimsson, Nonlinear photon trident versus double Compton scattering and resummation of one-step terms, Phys. Rev. D 102, 116008 (2020).

[24] A. Di Piazza, K.Z. Hatsagortsyan, and C. H. Keitel, Quantum Radiation Reaction Effects in Multiphoton Compton Scattering, Phys. Rev. Lett. 105, 220403 (2010). 
[25] D. Seipt, D. Del Sorbo, C. P. Ridgers, and A. G. R. Thomas, Theory of radiative electron polarization in strong laser fields, Phys. Rev. A 98, 023417 (2018).

[26] X. S. Geng, L. L. Ji, B. F. Shen, B. Feng, Z. Guo, Q. Q. Han, C. Y. Qin, N. W. Wang, W. Q. Wang, Y. T. Wu et al., Spindependent radiative deflection in the quantum radiationreaction regime, New J. Phys. 22, 013007 (2020).

[27] D. Seipt, C. P. Ridgers, D. Del Sorbo, and A. G. R. Thomas, Polarized QED cascades, New J. Phys. 23, 053025 (2021).

[28] V. Dinu and G. Torgrimsson, Approximating higher-order nonlinear QED processes with first-order building blocks, Phys. Rev. D 102, 016018 (2020).

[29] G. Torgrimsson, Loops and polarization in strong-field QED, New J. Phys. 23, 065001 (2021).

[30] Processes that correspond to (explicit or implicit) sums of loops only have been studied in e.g., Refs. [29,31-34].

[31] S. Meuren and A. Di Piazza, Quantum Electron SelfInteraction in a Strong Laser Field, Phys. Rev. Lett. 107, 260401 (2011).

[32] T. Podszus and A. Di Piazza, First-order strong-field QED processes including the damping of particle states, Phys. Rev. D 104, 016014 (2021).

[33] S. Bragin, S. Meuren, C. H. Keitel, and A. Di Piazza, High-Energy Vacuum Birefringence and Dichroism in an Ultrastrong Laser Field, Phys. Rev. Lett. 119, 250403 (2017).

[34] B. King and N. Elkina, Vacuum birefringence in highenergy laser-electron collisions, Phys. Rev. A 94, 062102 (2016).

[35] See Supplemental Material at http://link.aps.org/ supplemental/10.1103/PhysRevLett.127.111602 for details of the derivations of the recursive formula and the classical limits.

[36] F. Niel, C. Riconda, F. Amiranoff, R. Duclous, and M. Grech, From quantum to classical modeling of radiation reaction: A focus on stochasticity effects, Phys. Rev. E 97, 043209 (2018).

[37] M. Kh. Khokonov, Cascade processes of energy loss by emission of hard phonons, J. Exp. Theor. Phys. 99, 690 (2004).

[38] The fact that an $\alpha$ expansion would have to be resummed to obtain LL has recently been considered in Ref. [39].

[39] T. Heinzl, A. Ilderton, and B. King, Classical Resummation and Breakdown of Strong-Field QED, Phys. Rev. Lett. 127, 061601 (2021).

[40] A. Di Piazza, Exact solution of the Landau-Lifshitz equation in a plane wave, Lett. Math. Phys. 83, 305 (2008).

[41] H. Heintzmann and M. Grewing, Acceleration of charged particles and radiation-reaction in strong plane and spherical waves, Z. Phys. 251, 77 (1972).

[42] N. V. Elkina, A. M. Fedotov, I. Y. Kostyukov, M. V. Legkov, N. B. Narozhny, E. N. Nerush, and H. Ruhl, QED cascades induced by circularly polarized laser fields, Phys. Rev. ST Accel. Beams 14, 054401 (2011).

[43] V.S. Krivitsky and V. N. Tsytovich, Average radiation reaction force in quantum electrodynamics, Sov. Phys. Usp. 34, 250 (1991).

[44] A. Higuchi and G. D. R. Martin, The Lorentz-Dirac force from QED for linear acceleration, Phys. Rev. D 70, 081701(R) (2004).
[45] A. Higuchi and G. D. R. Martin, Radiation reaction on charged particles in three-dimensional motion in classical and quantum electrodynamics, Phys. Rev. D 73, 025019 (2006).

[46] A. Ilderton and G. Torgrimsson, Radiation reaction in strong field QED, Phys. Lett. B 725, 481 (2013).

[47] A. Ilderton and G. Torgrimsson, Radiation reaction from QED: Lightfront perturbation theory in a plane wave background, Phys. Rev. D 88, 025021 (2013).

[48] B. R. Holstein and J.F. Donoghue, Classical Physics and Quantum Loops, Phys. Rev. Lett. 93, 201602 (2004).

[49] S. V. Bulanova, T. Zh. Esirkepov, Y. Hayashi, M. Kando, H. Kiriyama, J. K. Koga, K. Kondo, H. Kotaki, A. S. Pirozhkov, S. S. Bulanov, A. G. Zhidkov, P. Chen, D. Neely, Y. Kato, N. B. Narozhny, and G. Korn, On the design of experiments for the study of extreme field limits in the interaction of laser with ultrarelativistic electron beam, Nucl. Instrum. Methods Phys. Res., Sect. A 660, 31 (2011).

[50] P. O. Kazinski and M. A. Shipulya, Asymptotics of physical solutions to the Lorentz-Dirac equation for a planar motion in constant electromagnetic fields, Phys. Rev. E 83, 066606 (2011).

[51] P. O. Kazinski, Radiation of de-excited electrons at large times in a strong electromagnetic plane wave, Ann. Phys. (Amsterdam) 339, 430 (2013).

[52] The probability that one of the emitted photons decays into a pair (trident pair production) scales as $e^{-16 /(3 \chi)}$ [15], which should be small.

[53] H. Mera, T. G. Pedersen, and B. K. Nikolić, Fast summation of divergent series and resurgent transseries from MeijerG approximants, Phys. Rev. D 97, 105027 (2018).

[54] G. Álvarez and H. J. Silverstone, A new method to sum divergent power series: Educated match, J. Phys. Commun. 1, 025005 (2017).

[55] G. Torgrimsson, Nonlinear trident in the high-energy limit: Nonlocality, Coulomb field and resummations, Phys. Rev. D 102, 096008 (2020).

[56] O. Costin and G. V. Dunne, Resurgent extrapolation: Rebuilding a function from asymptotic data. Painlevé I, J. Phys. A 52, 445205 (2019).

[57] O. Costin and G. V. Dunne, Physical resurgent extrapolation, Phys. Lett. B 808, 135627 (2020).

[58] E. Caliceti, M. Meyer-Hermann, P. Ribeca, A. Surzhykov, and U. Jentschura, From useful algorithms for slowly convergent series to physical predictions based on divergent perturbative expansions, Phys. Rep. 446, 1 (2007).

[59] A. Florio, Schwinger pair production from Padé-Borel reconstruction, Phys. Rev. D 101, 013007 (2020).

[60] G. V. Dunne and Z. Harris, On the higher loop EulerHeisenberg trans-series structure, Phys. Rev. D 103, 065015 (2021).

[61] G. A. Baker, Application of the Padé approximant method to the investigation of some magnetic properties of the Ising model, Phys. Rev. 124, 768 (1961).

[62] C. M. Bender and S. A. Orszag, Advanced Mathematical Methods for Scientists and Engineers, Asymptotic Methods and Perturbation Theory (Springer-Verlag, New York, 1999).

[63] H. Kleinert and V. Schulte-Frohlinde, Critical Properties of $\phi^{4}$-Theories (World Scientific, Singapore, 2001). 
[64] J. Zinn-Justin, Quantum Field Theory and Critical Phenomena, 4th ed. (Clarendon Press, Oxford, 2002).

[65] J. C. Le Guillou and J. Zinn-Justin, Critical exponents from field theory, Phys. Rev. B 21, 3976 (1980).

[66] V. I. Ritus, Effective Lagrange function of intense electromagnetic field in QED, in Proceedings of the Frontier Tests of QED and Physics of the Vacuum Sandanski, Bulgaria, 1998 (Heron Press, Sofia, 1998).

[67] I. K. Affleck, O. Alvarez, and N. S. Manton, Pair production at strong coupling in weak external fields, Nucl. Phys. B197, 509 (1982).

[68] I. Huet, M. Rausch De Traubenberg, and C. Schubert, Three-loop Euler-Heisenberg Lagrangian in $1+1$ QED, part 1: Single fermion-loop part, J. High Energy Phys. 03 (2019) 167.

[69] F. Karbstein, All-Loop Result for the Strong Magnetic Field Limit of the Heisenberg-Euler Effective Lagrangian, Phys. Rev. Lett. 122, 211602 (2019).

[70] A. A. Mironov, S. Meuren, and A. M. Fedotov, Resummation of QED radiative corrections in a strong constant crossed field, Phys. Rev. D 102, 053005 (2020).

[71] J. P. Edwards and A. Ilderton, Resummation of backgroundcollinear corrections in strong field QED, Phys. Rev. D 103, 016004 (2021).

[72] S. Zhang, Pre-acceleration from Landau-Lifshitz series, Prog. Theor. Exp. Phys. (2013), 123A01.

[73] H. Abramowicz, M. Altarelli, R. Aßmann, T. Behnke, Y. Benhammou, O. Borysov, M. Borysova, R. Brinkmann, F. Burkart, K. B. Üßer et al., Letter of Intent for the LUXE Experiment, arXiv:1909.00860.

[74] H. Abramowicz, U. Acosta, M. Altarelli, R. Aßmann, Z. Bai, T. Behnke, Y. Benhammou, T. Blackburn, S. Boogert, O. Borysov et al., Conceptual Design Report for the LUXE Experiment, arXiv:2102.02032.

[75] S. Meuren, P. H. Bucksbaum, N. J. Fisch, F. Fiúza, S. Glenzer, M. J. Hogan, K. Qu, D. A. Reis, G. White, and V. Yakimenko, On seminal HEDP research opportunities enabled by colocating multi-petawatt laser with highdensity electron Beams, arXiv:2002.10051. 